\title{
Irreversible Power and Radiation Resistance of Antennas in Magnetoionic Media
}

\author{
D. Walsh and $\mathrm{H}$. Weil \\ Radio Astronomy Observatory, University of Michigan, Ann Arbor, Mich. 48104, U.S.A.
}

(Received March 24, 1966)

\begin{abstract}
Certain objections, based on considerations of reversible as compared with irreversible power, which have been raised against conventional computations of the radiation resistance of antennas in magnetoplasma are shown to be without foundation. The inapplicability of this concept of revers. ible power to steady-state radiation is considered, and its connection with transient excitation is discussed.
\end{abstract}

The purpose of this note is to point out and correct certain misconceptions which appear in a recent paper concerning antenna radiation resistance computations [Lee and Papas, 1965]. In that paper it is stated that the time-average power, $P$, radiated by an antenna into a lossless gyroelectric medium is partially reversible and partially irreversible. Thus $P=P_{\text {rev }}+P_{\text {irr }}$ where $P_{\text {rev }}$ changes sign and $P_{\text {irr }}$ does not change sign under a time reversal transformation. The radiation resistance should be computed only from $P_{\text {irr }}$ since it is only this part of the power which is truly lost by the radiator. It is however claimed that the "conventional method" used by Weil and Walsh [1965] and by Staras [1964] for computation of radiation resistance of a small dipole in a lossless, cold magnetoplasma includes reversible power and hence is invalid. The formulas these authors used were derived by Kogelnik [1960] and by Kogelnik and Motz [1963]. It is further stated that these formulas "lead in general to an infinite value of the radiation resistance." This is, however, not the case. The formulas used by Weil and Walsh yield infinite values only when the index of refraction for propagation in the characteristic wave under consideration has infinite values (which for certain parameter values in a cold magnetoplasma will occur along a cone of directions about the static magnetic field). As these latter authors pointed out, the formulas they used are not valid under these conditions for several reasons. Moreover Staras demonstrated that by assuming a suitable current distribution over the antenna, a finite radiation resistance is obtained even for infinite index. Nevertheless Lee and Papas feel these results are basically incorrect due to inclusion of reversible power. By employing an analysis based on fields varying in a steady state as $\exp (-i \omega t)$ they derive the following formula for the time average of the irreversible power from a Hertz dipole located at the origin, $\mathbf{r}=0$ :

$P_{\text {irr }}=\frac{1}{4} \omega^{3} \mu_{0} \mathbf{p} \cdot \operatorname{Im}\left[\Gamma_{\text {out }}\left(0,0, \boldsymbol{B}_{0}\right)+\Gamma_{\text {out }}\left(0,0,-\boldsymbol{B}_{0}\right)\right] \cdot \boldsymbol{p}$ where $\boldsymbol{p}$ is the dipole moment (apparently taken to be real) and $\Gamma_{\text {out }}\left(\boldsymbol{r}, \boldsymbol{r}^{\prime}, \boldsymbol{B}_{0}\right)$ is the Green's dyadic which relates electric field $\boldsymbol{E}(\boldsymbol{r})$ to source current density $J\left(r^{\prime}\right)$ for outward going waves by

$$
\boldsymbol{E}_{\text {out }}\left(\boldsymbol{r}, \pm \boldsymbol{B}_{0}\right)=\omega \mu_{0} \int \Gamma_{\text {out }}\left(\boldsymbol{r}, \boldsymbol{r}^{\prime}, \pm \boldsymbol{B}_{0}\right) \cdot \boldsymbol{J}\left(\boldsymbol{r}^{\prime}\right) d V^{\prime}
$$

Lee and Papas state that the power expression obtained by the "conventional method" is

$$
P\left(\boldsymbol{B}_{0}\right)=\frac{1}{2} \omega^{3} \mu_{0} \boldsymbol{p} \cdot \operatorname{Im} \Gamma_{\text {out }}\left(0,0, \boldsymbol{B}_{0}\right) \cdot \boldsymbol{p}
$$

and that $P\left(\boldsymbol{B}_{0}\right) \neq P_{\text {irr- }} \quad$ Equation (3) is certainly equivalent to the formula used by Weil and Walsh [1964] for an electric dipole and an analogous formula was used for an elementary loop [Weil and Walsh, 1965]. However, contrary to the statement of Lee and Papas, $P\left(\boldsymbol{B}_{0}\right)=P_{\mathrm{irr}}$. An examination of the explicit formula for $\Gamma\left(0,0, \boldsymbol{B}_{0}\right)[$ Kogelnik, 1960] shows that its imaginary part has the form

$$
\operatorname{Im} \Gamma_{\text {out }}\left(0,0, \pm B_{0}\right)=\left(\begin{array}{ccc}
\gamma_{11} & \pm \gamma_{12} & \pm \gamma_{13} \\
\mp \gamma_{12} & \gamma_{11} & \pm \gamma_{23} \\
\mp \gamma_{13} & \mp \gamma_{23} & \gamma_{33}
\end{array}\right)
$$

Hence, if $\boldsymbol{p}=\left(p_{1}, p_{2}, p_{3}\right)$,

$P\left( \pm \boldsymbol{B}_{0}\right)=\frac{1}{2} \quad \omega^{3} \mu_{0}\left[\gamma_{13}\left(\left|p_{1}\right|^{2}+\left|p_{2}\right|^{2}\right)+\gamma_{33}\left|p_{3}\right|^{2}\right]=P_{\mathrm{irr}}$.

The effect of the sign of $\boldsymbol{B}_{0}$ appears only in the offdiagonal terms of $\Gamma\left(0,0, B_{0}\right)$ which do not contribute to the bilinear form. Hence, whether this effect is cancelled "in advance," as it is by using $\operatorname{Im}\left[\Gamma_{\text {out }}(0,0\right.$, $\left.\left.\boldsymbol{B}_{0}\right)+\Gamma_{\text {out }}\left(0,0,-\boldsymbol{B}_{0}\right)\right]$, or is not, the result is seen, on a purely formal basis, to be the same.

One might say that in this case the theory of Lee and Papas indicates that for a Hertz dipole there is no reversible real power. However, the meaningfulness of the concept itself is questionable on physical grounds for steady-state radiation in an unbounded 
medium. In the following we illustrate by example why this is so and consider the applicability of the concept of reversible power under transient conditions. Consider a short, but not infinitesimal, electric dipole in vacuum excited by a voltage $V_{0} \exp \left(-i \omega_{0} t\right)$ turned on at time $i=0$. Once the transients have effectively died out the impedance $R-i X$ will be dominantly reactive $(|X|>>R)$. The average radiated power, $\frac{1}{2}|I|^{2} R$, may then be calculated by integrating the Poynting vector over a closed surface surrounding the antenna. Only the "far field," i.e., field components varying as $r^{-1}$, where $r$ is distance from the dipole, contributes to this average which represents in fact irreversible power. The "near field," consisting of the field components varying as higher powers of $r^{-1}$ is, to good approximation, that of a capacitance, $C$. For the real applied voltage, $V_{0} \cos \omega_{0} t$, the energy in the near field is, therefore

$$
E \cong \frac{1}{4} C V_{0}^{2}\left(1+\cos 2 \omega_{0} t\right)
$$

and its rate of change is

$$
\frac{d E}{d t}=-\frac{1}{2} \omega_{0} C V_{0}^{2} \sin 2 \omega_{0} t
$$

Thus $d E / d t$ has zero average value and represents reactive power or energy exchange between the exciting source and the near field. However, in addition to this oscillatory reactive energy, $E$ has a constant term contributing nothing to energy exchange but representing nonzero average energy stored in the near field. This average energy is not detectable by measurements made at the antenna terminals in the steady state. It was supplied by the source during the transient after excitation commenced and may be withdrawn by a suitable termination after the excitation is stopped, as may the energy stored in any capacitor. In this sense the energy exchange during the transient stages is partially reversible. The quantity

$$
-\int \boldsymbol{J} \cdot \boldsymbol{E}_{\mathrm{ret}} d V
$$

where $\boldsymbol{E}_{\text {ret }}$ is the retarded electric field due to the current density distribution, $\boldsymbol{J}$, represents the rate at which work is done on the electromagnetic field and is equal at all times to the sum of reversible and irreversible power. In summary, during the initial transient, both reversible and irreversible power are supplied to the field, but in the steady state the average power supplied is purely irreversible.

A physically related problem is that of radiation by an accelerated electron. Lee and Papas cite the analysis of Schwinger [1949], who considered the rate at which the electron does work on the electromagnetic field, given by (8), and divided it into two parts. One remained unchanged on reversing the positive sense of time, and Schwinger recognized it as "resistive power" describing the rate of irreversible energy transfer to the electromagnetic field, which represented the desired rate of radiation. The other part changed sign on reversing the positive sense of time, and Schwinger recognized it as "reactive power" describing the rate at which the electron stores energy in the electromagnetic field. This is an inertial effect, related to the momentum and kinetic energy of the electron. The resistive power depends only on field components varying as $r^{-1}$, and Schwinger's results may be derived by considering only such components [Panofsky and Phillips, 1962]. These fields depend linearly on the acceleration of the electron. The reactive power depends only on the speed, and is related to those field components varying as higher powers of $r^{-1}$. Panofsky and Phillips, in fact, recognize these "convective" field components as being analogous to the quasi-static or induction field connected with radiation from variable current or charge systems. If the speed of an electron is increased so that its momentum and kinetic energy are increased, energy is supplied to these fields, and if the speed is decreased, energy is withdrawn from them; on the other hand, there is an irreversible loss of energy to the $r^{-1}$ fields during any change of velocity. The analogy between reversible and irreversible power associated with an accelerated electron and with an antenna is complete. For example, if an electron executes steady-state harmonic motion, the timeaverage reversible power is zero even though the average energy in the reactive fields is nonzero and was supplied during the starting transient, exactly as in the above discussion of a short dipole antenna.

The reversible power discussed above exists in vacuum; it has nothing to do with the presence of a static magnetic field. Similarly, in the case of an antenna in a plasma, reversible power exists in both transient and steady-state conditions, regardless of the presence or absence of a static magnetic field, but has zero time-average value under steady-state conditions. In those zases where the refractive index is always finite, the starting transient presumably becomes negligible after sufficient time, and the steady-state computations of radiation resistance are correct. Time average power has its usual meaning and has nothing to do with reversible power. However, under conditions where the refractive index may become infinite, Walsh and Haddock [1965] point out that "it is possible that infinite time is required to supply all the energy that would be required to achieve a steady state, even though this energy is supplied to the near field which one expects to be essentially reactive. This behavior might be described as an 'extended transient,' and may result in an apparently resistive impedance even though reactive fields are being built up." The reactive fields referred to by Walsh and Haddock are clearly those associated with reversible power. 
In conclusion, it appears that the results of Weil and Walsh [1964, 1965] are valid except (as recognized by them) under conditions where the refractive index may be infinite. Reversible power has zero time-average value except under transient conditions, and any attempt to calculate it must take this into account.

This work was supported by the National Aeronautics and Space Administration under Grant NsG572 .

\section{References}

Kogelnik, H. (1960), The radiation resistance of an elementary dipole in anisotropic plasmas, Proc. Fourth Intern. Conf. on Ionization Phenomena in Gases (North-Holland Publishing Co., Amsterdam).

Kogelnik, H. and H. Motz (1963), Electromagnetic radiation from sources embedded in an infinite anisotropic medium and the significance of the Poynting vector, Proc. Symp. on Electromagnetic Theory and Antennae (Pergamon Press, New York, N.Y.).
Lee, K. S. H. and C. H. Papas (1965), Irreversible power and radiation resistance of antennas in anisotropic ionized gases, Radio Sci. J. Res. NBS 69D, No. 10, 1313-1320.

Panofsky, W. K. H. and M. Phillips (1962), Classical Electricity and Magnetism, 2nd ed., ch. 20 (Addison-Wesley, Reading, Mass.).

Schwinger, J. (1949), On the classical radiation of accelerated elec trons, Phys. Rev. 75, No. 12, 1912-1925.

Staras, $H$. (1964), The impedance of an electric dipole in a cold anisotropic medium, IEEE Trans. Ant. Prop. AP-12, No. 6, 695702.

Walsh, D. and F. T. Haddock (1965), Antenna impedance in a plasma: problems relevant to radio astronomy measurements from space vehicles, Ann. Astrophys. 28, No. 3, 605-615. Reprinted in Astronomical Observations from Space Vehicles (Proc. of IAU Symp. No. 23, Aug. 1964), J. L. Steinberg, ed., Ann. Astrophys. Meudon (S-et-O), France.

Weil, H. and D. Walsh (1965), Radiation resistance of an elementary loop antenna in a magnetoionic medium, IEEE Trans. Ant. Prop. AP-13, No. 1, 21-27.

Weil, H. and D. Walsh (1964), Radiation resistance of an electric dipole in a magnetoionic medium, IEEE Trans. Ant. Prop. AP-12, No. 3, 297-304.

\title{
On Walsh and Weil's Defense of the Conventional Method
}

\author{
K. S. H. Lee and C. H. Papas \\ California Institute of Technology, Pasadena, Calif. 91109, U.S.A.
}

(Received April 18, 1966)

As we [Lee and Papas, 1965] have pointed out, the steady-state, time-average power $P$ emitted by an antenna in an anisotropic medium has two parts, an irreversible part $P_{\text {irr }}$ and a reversible part $P_{\text {rev }}$. That is, $P=P_{\text {irr }}+P_{\text {rev }}$. It is our contention that true radiated power is given by $P_{\text {irr }}$ and not necessarily by $P$. In special cases where $P_{\text {rev }}=0$, e.g., the case where the antenna is in a vacuum or the case where the antenna is in a homogeneous, isotropic, lossless medium, it makes no difference whether one uses $P$ or $P_{\text {irr }}$ as the measure of radiated power. However, if the medium surrounding the antenna is gyroelectric, there is no reason to believe that $P_{\text {rev }}=0$, and one must be careful to account for the difference between $P$ and $P_{\text {irr }}$.

To be safe, one should find the radiated power by calculating $P_{\text {irr }}$ directly, because $P_{\text {irr }}$ always gives the radiated power regardless of whether $P_{\text {rev }}$ is or is not zero. On the other hand, $P$ gives the radiated power only when $P_{\text {rev }}=0$.

Walsh and Weil [1966] seem to believe that $P_{\text {rev }}$ is always zero, even in a gyroelectric medium. They attempt to prove their point, but their "proof" is not convincing; it suffers from the same weakness as Staras' "proof." This weakness has already been placed in evidence by us [Lee and Papas, 1966].

To physically justify their conjecture that $P_{\text {rev }}=0$ always, Walsh and Weil [1966] consider the trivial case of an antenna in vacuum. They show that in this vacuum case $P_{\text {rev }}=0$. But what bearing this result has on the discussion is somewhat of a mystery. We have repeatedly stated that $P_{\text {rev }}=0$ in the vacuum case, so there is no disagreement in this case. The physical arguments that Walsh and Weil invoke do not apply to the case of a gyroelectric medium and in no way indicate that $P_{\text {rev }}$ is identically zero in the gyroelectric case.

\section{References}

Lee, K. S. H. and C. H. Papas (1965), Irreversible power and radiation resistance of antennas in anisotropic ionized gases, Radio Sci. J. Res. NBS 69D, No. 10, 1313-1320.

Lee, K. S. H. and C. H. Papas (1966), A further explanation of the new theory of antenna radiation with particular reference to uhiaxial media, Radio Sci. 1 (New Series), No. 9, 1020-1023.

Walsh, D. and H. Weil (1966), Irreversible power and radiation resistance of antennas in magnetoionic media, Radio Sci. 1 (New Series), No. 9, 1025-1027.

(Paper 1-9-109) 